archives-ouvertes

\title{
Ion velocity analysis of rotating structures in a magnetic linear plasma device
}

\author{
N. Claire, A. Escarguel, C. Rebont, F. Doveil
}

\section{To cite this version:}

N. Claire, A. Escarguel, C. Rebont, F. Doveil. Ion velocity analysis of rotating structures in a magnetic linear plasma device. Physics of Plasmas, American Institute of Physics, 2018, 25 (6), pp.061203. 10.1063/1.5019448. hal-02103315

\section{HAL Id: hal-02103315 https://hal-amu.archives-ouvertes.fr/hal-02103315}

Submitted on 18 Apr 2019

HAL is a multi-disciplinary open access archive for the deposit and dissemination of scientific research documents, whether they are published or not. The documents may come from teaching and research institutions in France or abroad, or from public or private research centers.
L'archive ouverte pluridisciplinaire HAL, est destinée au dépôt et à la diffusion de documents scientifiques de niveau recherche, publiés ou non, émanant des établissements d'enseignement et de recherche français ou étrangers, des laboratoires publics ou privés. 


\title{
Ion velocity analysis of rotating structures in a magnetic linear plasma device
}

\author{
N. Claire, ${ }^{\text {a) }}$ A. Escarguel, ${ }^{\text {b) }}$ C. Rebont, and F. Doveil ${ }^{\text {() }}$ \\ Aix Marseille Univ, CNRS, PIIM, Marseille, France
}

\begin{abstract}
The MISTRAL device is designed to produce a linear magnetized plasma column. It has been used a few years ago to study a nonlinear low frequency instability exhibiting an azimuthal number $m=2$. By changing the experimental configuration of MISTRAL, this work shows experimental results on an $m=1$ rotating instability with strongly different behavior. The spatio-temporal evolution of the ion velocity distribution function given by a laser-induced fluorescence diagnostic is measured to infer the radial and azimuthal velocities, ion fluxes, and electric fields. The naive image of a plasma exhibiting a global rotation is again invalidated in this $m=1$ mode but in a different way. Contrary to the $m=2$ mode, the rotation frequency of the instability is lower than the ion cyclotron frequency and ions exhibit a complex behavior with a radial outward flux inside the unstable arm and azimuthal ion fluxes always directed toward the unstable arm. The azimuthal ion velocity is close to zero inside the ionization region, whereas the radial ion velocity grows linearly with radius. The radial electric field is oriented inward inside the unstable arm and outward outside. An axial velocity perturbation is also present, indicating that contrary to the $m=2$ mode, the $m=1$ mode is not a flute mode. These results cannot be easily interpreted with existing theories.
\end{abstract}

https://doi.org/10.1063/1.5019448

\section{INTRODUCTION}

The presence of a magnetic field $\vec{B}$ perpendicular to an electric field $\vec{E}$ in a plasma leads to charged particle drift in the $\vec{E} \times \vec{B}$ direction. This situation is very favorable for the triggering of instabilities, leading to anomalous plasma transport across the magnetic field. This is a crucial question of plasma physics, which has attracted much attention in the context of fusion plasmas, but little is known about anomalous transport in low temperature discharge devices (Hall thrusters, negative ion sources, Penning discharges, magnetrons, mass separation of nuclear waste, etc.) where the presence of weakly or non-magnetized ions and of collisions with neutrals leads to specific phenomena. Strongly nonlinear rotating structures can develop in such configurations, exhibiting a large density and potential perturbation with angular frequency close to the ion cyclotron frequency. Depending on the experimental conditions, it has been shown that radial regular rotating structures with azimuthal numbers $m=1$ and $m=2$ can be observed on the MISTRAL plasma device. ${ }^{1-4}$ The transition from regular to unstable or turbulent regimes can be controlled by biasing the end plates of the plasma column. In 2011, a detailed study of such instability with two rotating symmetric arms $(m=2)$ was investigated experimentally on MISTRAL, ${ }^{5}$ with emphasis on the ion velocity distribution function (IVDF). By changing the experimental conditions on MISTRAL with a conducting cylinder surrounding the plasma column, we present in this work a complementary study of an $m=1$ strongly nonlinear coherent structure using the same Laser Induced Fluorescence (LIF) diagnostic. ${ }^{6,7}$ Time-resolved LIF is

\footnotetext{
${ }^{\text {a)} E l e c t r o n i c ~ m a i l: ~ n i c o l a s . c l a i r e @ u n i v-a m u . f r ~}$

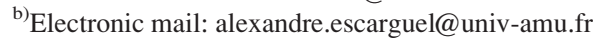

${ }^{\mathrm{c}}$ Electronic mail: fabrice.doveil@univ-amu.fr
}

applied to the investigation of temporal and spatial evolution of the IVDF inside and outside the ionization region. In comparison with the $m=2$ case, the spatiotemporal evolution of the IVDF exhibits very different features.

\section{EXPERIMENTAL APPARATUS}

The MISTRAL device is composed of a large source chamber and a cylindrical study chamber. Energetic ionizing electrons are created in the source chamber, with typical energy around $40 \mathrm{eV}$, and are accelerated toward the grounded anode. As shown in Fig. 1, a magnetic multipolar structure increases the confinement time of the ionizing electrons and allows their radially homogeneous injection inside the cylindrical study chamber.

The plasma study is done in this second chamber. It allows the creation of a linear plasma column confined by an axial magnetic field. It is $1 \mathrm{~m}$ in length and $40 \mathrm{~cm}$ in diameter. The argon gas pressure is $9 \times 10^{-4}$ mbar. The uniform magnetic field of $16 \mathrm{mT}$ is produced by a set of 19 coils. A metallic diaphragm limiter with a centered circular $10 \mathrm{~cm}$ aperture is placed between the source and the study chamber. For the experiments reported here, two grounded halfcylinders have been installed around the plasma column to measure the time evolution of the radially collected current. Therefore, the distance between the core plasma and the vessel is less than for the configuration in which an instability with azimuthal number $m=2$ has been previously studied. ${ }^{5}$ Consequently, the radial current evacuation from plasma to ground is easier. The linear magnetized plasma column is axially delimited by two grids at the floating potential, one between the source and the study chamber and the other one at the end of the column.

The equilibrium of the plasma column is determined by the current of incoming ionizing electrons and the collection 


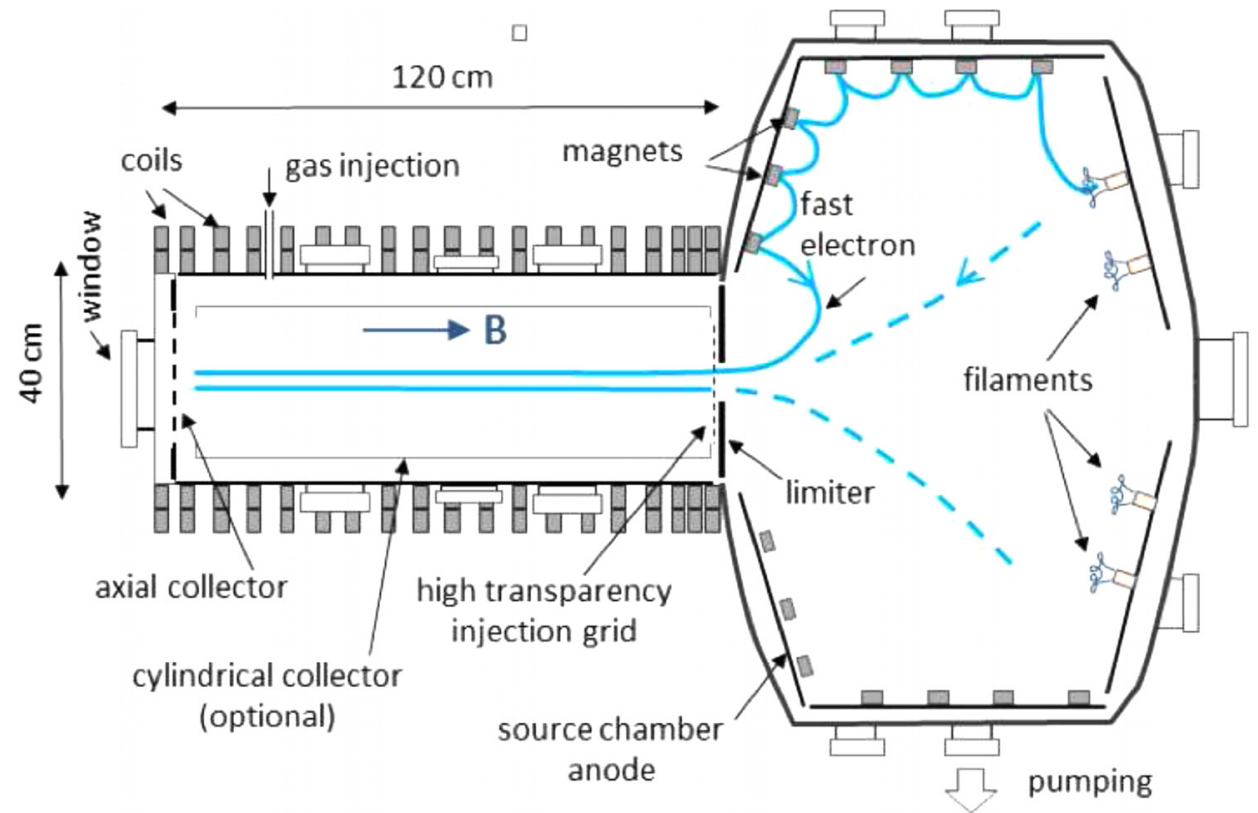

of charged particles on the separating and collecting grids, and on the vessel or the two half-cylinders. The potential difference between the central plasma column and the grounded collecting half-cylinders gives rise to an inward radial electric field, leading to a possible $\vec{E} \times \vec{B}$ rotation of ions and electrons. Nonlinear rotating structures around a plasma column have been detected a long time ago in magnetized plasmas. ${ }^{8}$ Analytical studies have exhibited the main characteristics 9,10 of these structures existing when a sheared radial electric field is established. The structure of the instability has been obtained with probes, ${ }^{11}$ ultrafast imaging, ${ }^{12}$ and spectroscopy. ${ }^{4}$ A kinetic description of the unstable low-frequency waves in a magnetized plasma column under rotation induced by the injection of energetic electrons has been proposed. ${ }^{13}$ More recently, PIC-MCC (Particle-In-Cell Monte Carlo Collisions) simulations have shown some important features of $\vec{E} \times \vec{B}$ configurations rotating instabilities. ${ }^{14}$

The plasma parameters are the electronic density $n_{e}$ $\approx 10^{8} \mathrm{~cm}^{-3}$, the ion cyclotronic frequency $f_{c i}=6 \mathrm{kHz}$, the electronic cyclotronic frequency $f_{c e}=400 \mathrm{MHz}$, the electronic Larmor radius $\rho_{c e}=0.3 \mathrm{~mm}$, the ion Larmor radius $\rho_{c i}=12.8 \mathrm{~mm}$, the electronic temperature $T_{e} \approx 3 \mathrm{eV}$, the ion temperature $T_{i} \approx 0.1 \mathrm{eV}$, and the plasma potential $\Phi_{\text {plasma }}=10 \mathrm{~V}$. In these conditions (grounded half cylinders and grids at floating potential), a strongly non-linear rotating coherent $m=1$ mode develops in the central plasma at $5 \mathrm{kHz}$ with one spiral arm and expands out in the scrape-off layer behind the entrance limiter. The LIF diagnostic ${ }^{6}$ uses a tunable dye laser chopped by an acousto-optic modulator and pumped by a continuous DPSS Verdi laser. The spectral purity of the dye laser is very high $(0.5 \mathrm{MHz})$, and the central frequency is electronically tunable. Three Argon II atomic levels are used: the $3 d_{2} G^{9 / 2}$ metastable level is excited at $611 \mathrm{~nm}$ to the $4 p_{2} F^{7 / 2}$ level whose decay at $460.9 \mathrm{~nm}$ to the $3 s_{2} G^{9 / 2}$ level is observed. With this method, the IVDF along the laser beam can be recorded with a good spatial resolution. ${ }^{15}$ The laser beam is fired vertically at different plasma radiuses from $r=1$ to $6 \mathrm{~cm}$, the entrance diaphragm radius
FIG. 1. Sketch of the MISTRAL experimental device. being $r=5 \mathrm{~cm}$. Because of too noisy data, no acquisition has been done for $r>6 \mathrm{~cm}$. The fluorescence radiation emitted by the probed metastable ions is collected in a direction perpendicular to the laser beam by an optical system whose aperture sets the axial radial and azimuthal resolution at $0.8 \mathrm{~cm}$. This value is close to the ion Larmor radius with thermal velocity $v_{t h i}=450 \mathrm{~m} / \mathrm{s}$. The fluorescence radiation is detected by a photomultiplier tube coupled to a $1 \mathrm{~nm}$ bandpass optical filter. A multi-channel scaler $^{16}$ records the counting rate of events. With repetitive measurements, the scan in laser frequency is synchronized with the rotation of the structure inside the plasma and multiple scans are summed up. The temporal resolution is $10 \mu \mathrm{s}$ and the duration of one record is about $20 \mathrm{~min}$.

\section{III. $m=1$ MODE RESULTS}

When separating and collecting grids are grounded, no rotating structure is present. A $m=1$ mode is obtained for the collecting and separating grids at floating potential and the half cylinders grounded as shown on Fig. 1.

Figure 2 shows the time evolution of the plasma light images separated by $25 \mu \mathrm{s}$ in the shadow of the limiter. This result is obtained with a synchronized intensified camera looking at the end of the plasma column exhibiting a $m=1$ plasma ejection mode at $\nu=5 \mathrm{kHz}$. To avoid saturation of the camera, the plasma bulk light emission is occulted by a mask. This series of pictures shows clearly the rotation of the $m=1$ structure. $^{4}$

In this configuration, the charged particles flux cannot be evacuated by the floating grids placed at the two ends of the plasma column. The only possibility is the radial evacuation through the two half-cylinders. Then, this experimental configuration favors the radial transport across the magnetic field with respect to the $m=2$ mode configuration. Moreover, we note that it is much easier to obtain nonlinear modes in rotation with the presence of the two half-cylinders than without. 


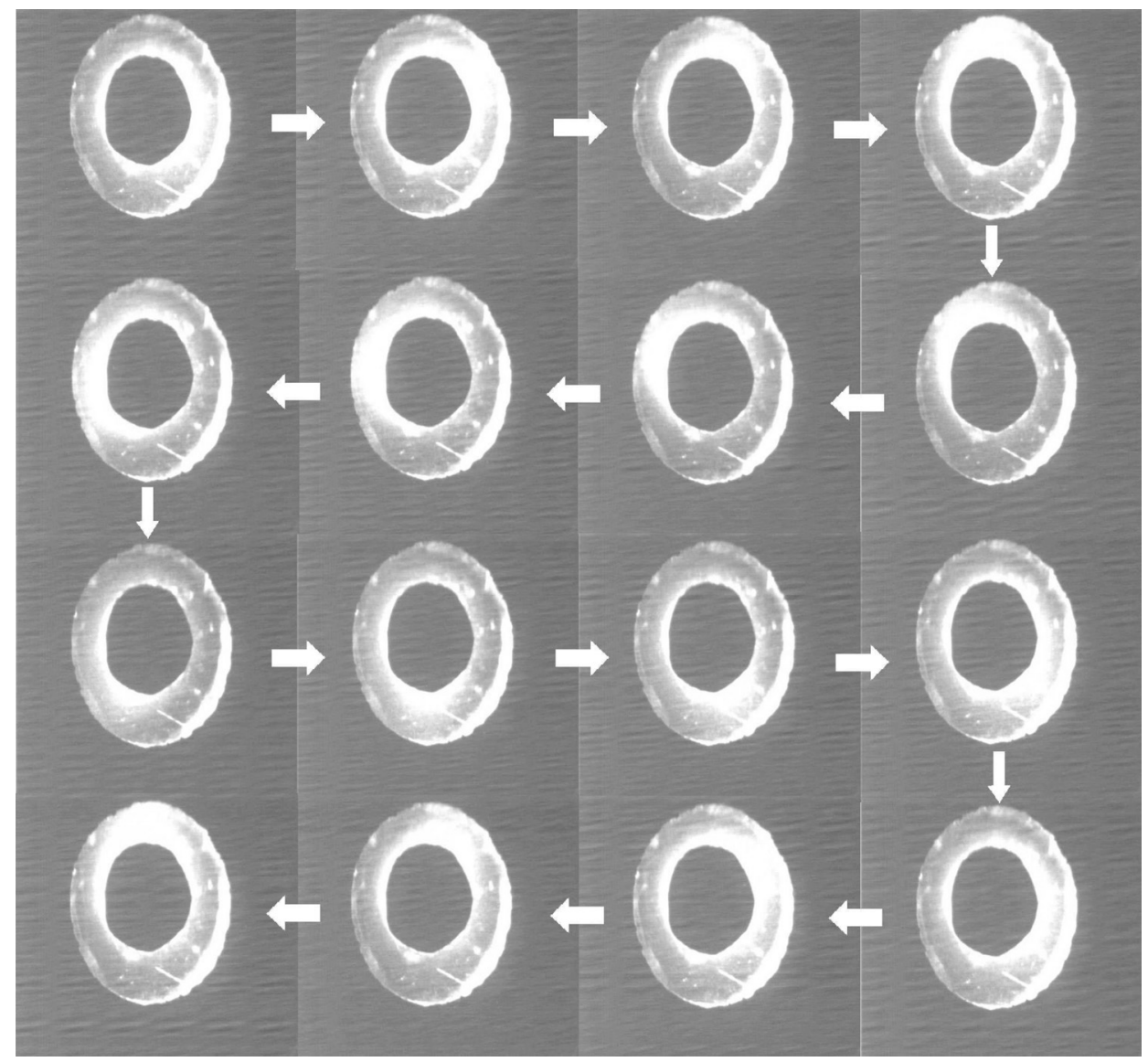

FIG. 2. Images from an intensified camera, plasma bulk emission is occulted by a mask.

Plasma ejection occurs in the form of one spiral arm in this particular MISTRAL configuration. The main control parameters are the collecting and separating grids potentials. The other device parameters (discharge biasing, pressure, ...) only change the value of the rotation frequency of the structure. An $m=2$ mode, similar to the one previously studied, ${ }^{5}$ can also be obtained in this half-cylinders MISTRAL configuration when separating and collecting grids are biased at a different potential, for example, $\Phi_{\text {col }}=20 \mathrm{~V}$ and $\Phi_{\text {sep }}$ $=2 \mathrm{~V}$. Spatial/time resolved IVDF of Argon ions was measured in the plasma. From these data, the $2 \mathrm{D}$ ion velocity $\vec{v}$ and electric field $\vec{E}$ have been calculated in the same way as for the $m=2$ study, $^{5}$ for radius varying from $r=1 \mathrm{~cm}$ to $r=6 \mathrm{~cm}$ with a $1 \mathrm{~cm}$ step. The spatial and temporal resolutions are equal to $8 \mathrm{~mm}$ and $10 \mu \mathrm{s}$, respectively. The $r=6 \mathrm{~cm}$ measurements correspond to $1 \mathrm{~cm}$ outside the ionization region. Positive azimuthal fluxes, velocities, and electric fields are oriented in the trigonometrical sense which is the direction of the structure rotation. Radial fluxes, velocities, and electric fields are positive when directed outward the plasma column.

Figure 3 shows a typical radial time-resolved IVDF record at $r=5 \mathrm{~cm}$ from the center and at a time corresponding to the maximum density perturbation. Close to a Maxwellian distribution (except at far wings), its total thermal velocity $v_{t h r}$ has a value of $810 \mathrm{~m} / \mathrm{s}$ for this point at this particular time in the MISTRAL device. This value is close to the value classically measured for the ions in MISTRAL. But the time evolution of $v_{t h r}$ at $r=5 \mathrm{~cm}$ exhibits a particular shape, as shown in Fig. 4: it is minimum at the maximum density perturbation and 4 times bigger at the minimum density perturbation. This thermal velocity behavior is also present on the azimuthal records end for any radius. Thus ions clearly exhibit a higher temperature outside the rotating structure. Using the same reconstruction as in the paper by Rebont et al. ${ }^{5}$ for this kind of rotating periodic structure, a measurement by LIF of IVDF along the radial direction at one position gives a measurement of IVDF along the azimuthal direction at a position angularly shifted by is $\pi / 2$ at

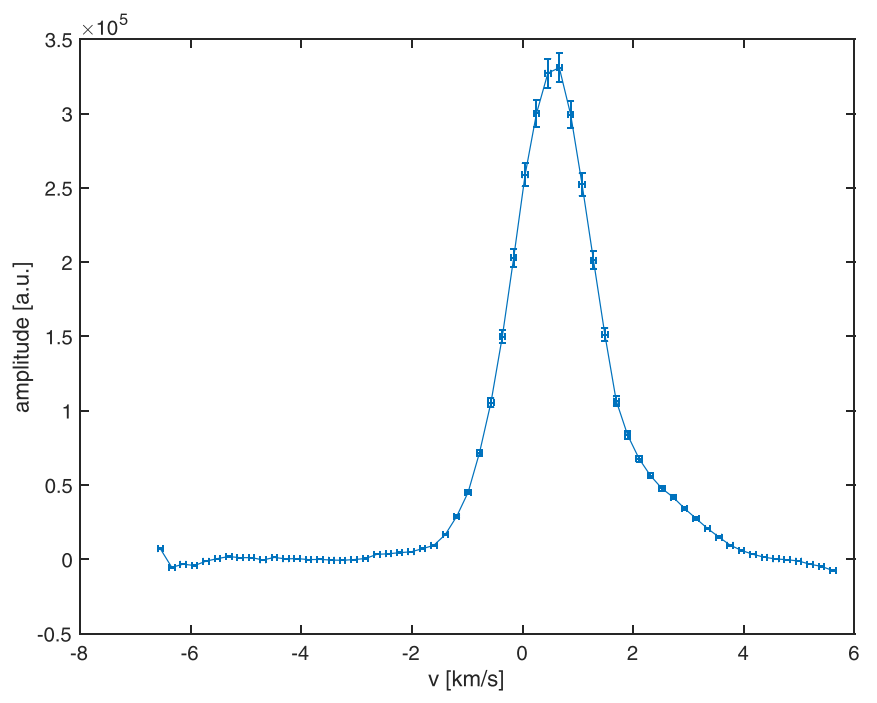

FIG. 3. Typical radial IVDF record at $r=5 \mathrm{~cm}$ from the center and at the time of maximum density perturbation. 


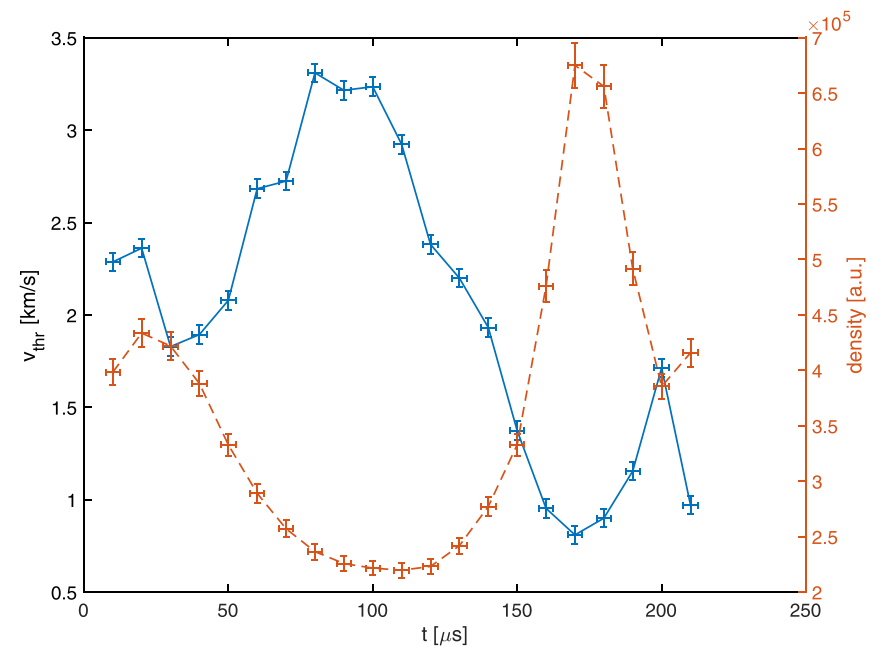

FIG. 4. Plasma density (dashed line) and total radial thermal velocity $v_{t h r}$ (continuous line) at $r=5 \mathrm{~cm}$ as a function of time.

the same radius. Time is then synchronized between the radial and reconstructed azimuthal measurements, assuming that the maximum density perturbation must occur at the same time for both measurements.

(a)

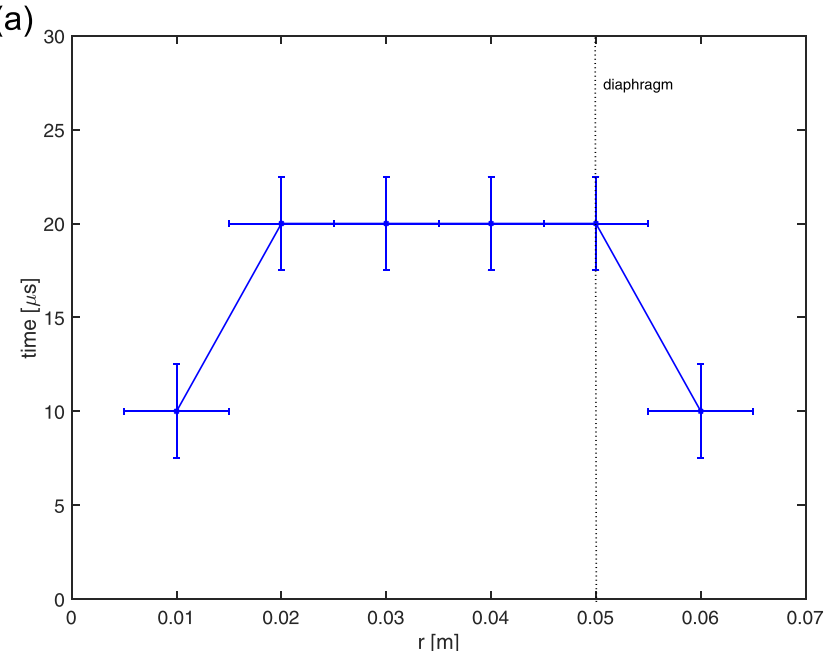

(b)

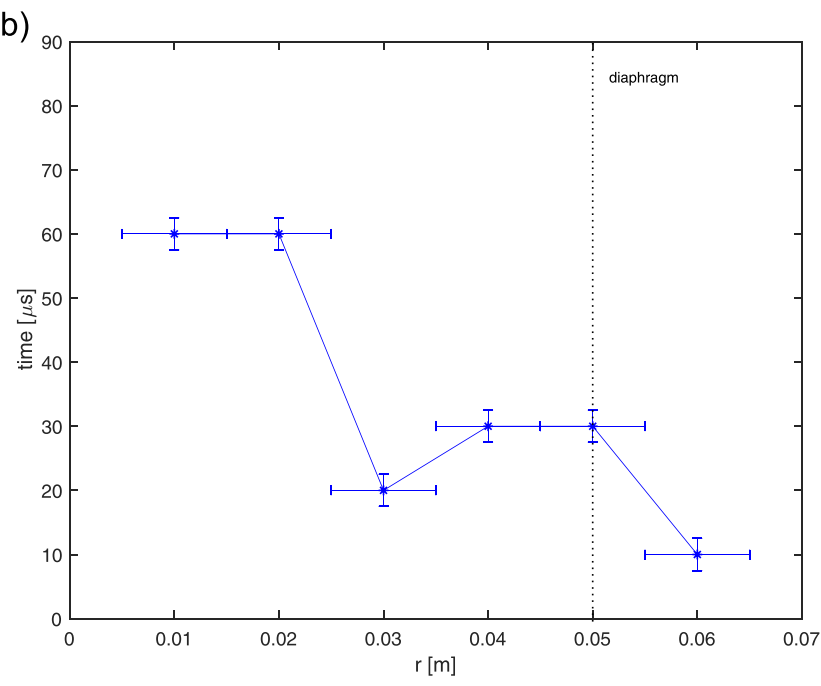

FIG. 5. Radial variation of the time delay between the maximum of the ionic density and the maximum of the azimuthal (a) and radial (b) fluid velocities.

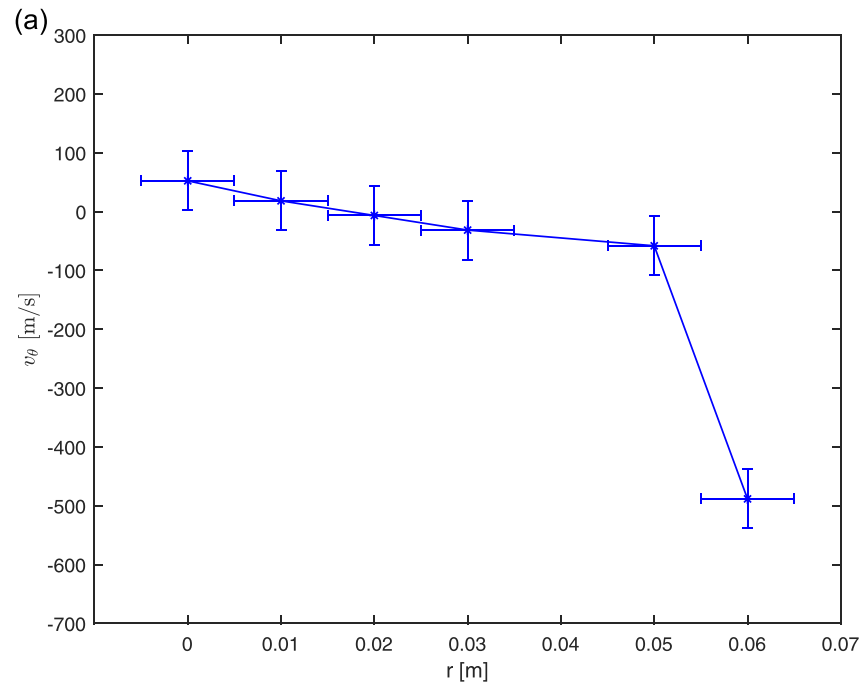

(b)

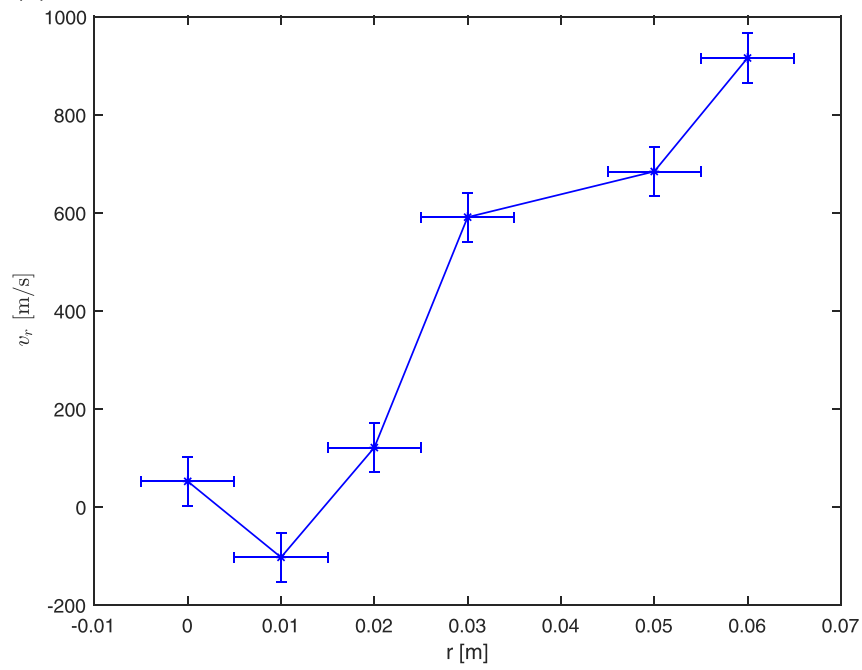

FIG. 6. Radial variations of the ionic azimuthal (a) and radial (b) fluid velocities at the density maximum.

In Fig. 5, the radial variation of the time delay between the ion density maximum and the azimuthal and radial maximum fluid velocities is presented. The azimuthal time delay is approximately constant and close to $20 \mu$ s (corresponding to a $\pi / 5$ phase shift), but the radial time delay strongly decreases with radius: inside the ionization region, the maximum phase shift is $3 \pi / 5(60 \mu \mathrm{s})$, then decreases to half of this value, and vanishes outside. Let us recall that theory predicts a null (resp. $\pi / 2$ ) phase shift for a flute mode (resp. a drift wave). ${ }^{17}$

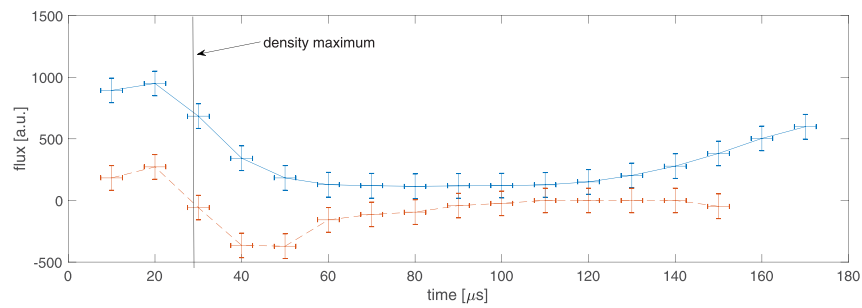

FIG. 7. Temporal evolutions of the azimuthal (dashed line) and radial (continuous line) ionic fluxes at $r=5 \mathrm{~cm}$. The vertical line represents the density maximum. 


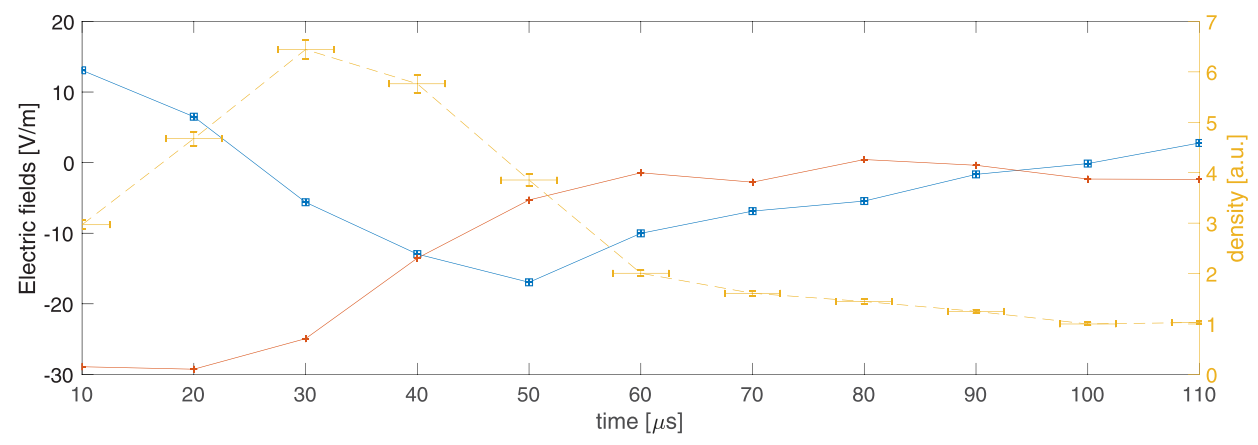

FIG. 8. Temporal evolutions of the density (dashed line), the azimuthal (continuous line) and radial (squares) electric fields at $r=5 \mathrm{~cm}$.

In Fig. 6, the azimuthal and radial fluid velocities are presented versus radius for the time corresponding to the maximum density perturbation in the arm. Except outside the ionization region, the azimuthal fluid velocity $v_{\theta}$ is very low and decreases slightly with radius, even if a small misalignment is possible and could explain the negative values. The radial fluid velocity increases approximately linearly with radius as used in an earlier theoretical approach, ${ }^{10}$ following the relation $v_{r}=A . r$ with $A=20340 \mathrm{~s}^{-1}$. These results show that, at the density maximum inside the arms, ions do not rotate with it.

Figure 7 shows the temporal evolution of the radial and azimuthal fluxes, $\Gamma_{r}=n \cdot v_{r}$ and $\Gamma_{\theta}=n . v_{\theta}$ for $r=5 \mathrm{~cm}$, the vertical line corresponding to the density maximum. Note that a complete period of the perturbation is not shown. Inside the arm, fluxes behaviors are similar for all radiuses: ions are radially ejected outward (positive radial flux around the density maximum). In the azimuthal direction, the flux changes its sign at the density maximum (positive before and negative after the maximum). This means that at the periphery of the unstable arm, ions always penetrate azimuthally inside it.

The azimuthal $E_{\theta}$ and radial $E_{r}$ electric fields have been calculated from the fluid equations as in the previous work on the $m=2$ instability. ${ }^{5}$ Figure 8 gives the temporal evolution of the ion density $n_{i}$ and of the azimuthal $E_{\theta}$ and radial $E_{r}$ electric fields at position $r=5 \mathrm{~cm} . E_{r}$ changes its sign around the density maximum, pointing outward before it. No error bar is plotted since electric fields are derivated from a theoretical fluid model. ${ }^{5}$ The norm of the electric field vector increases with radius, from $10 \mathrm{Vm}^{-1}$ close to the plasma column center to $40 \mathrm{Vm}^{-1}$ at $r=6 \mathrm{~cm}$ outside the ionization region.

In Fig. 9, the time evolution of the axial ion fluid velocity and density is shown. Unlike the $m=2$ case, ${ }^{5}$ an axial velocity perturbation is clearly present. The $m=1$ rotating structure

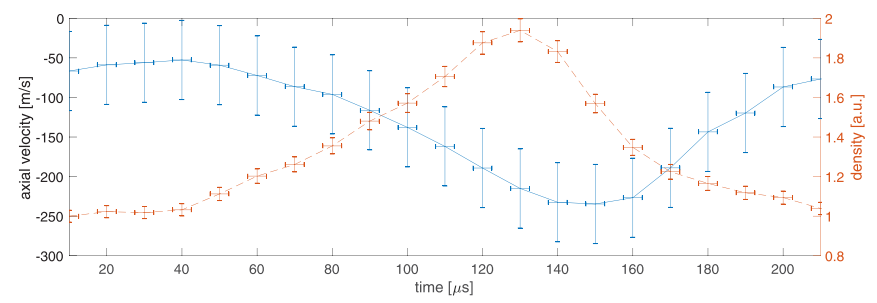

FIG. 9. Temporal evolutions of axial ion fluid velocity (continuous line) and density (dashed line). in the linear magnetized plasma MISTRAL is not a flute mode characterized by a revolution symmetry and an identical radial perturbation expansion all along the column. The axial velocity is always directed toward the end grid with a maximum amplitude close to the maximum density perturbation.

\section{COMPARISON BETWEEN $m=1$ AND $m=2$ MODE}

The $m=2$ and $m=1$ modes show very different behaviors. Obviously, $m=2$ is symmetric, whereas $m=1$ is not. Indeed, $m=2$ mode presents more symmetry than the $m=1$ mode, whereas the center of the plasma column of the $m=1$ mode spatially oscillates around the column center with the arm rotation. ${ }^{18}$ The ion cyclotronic frequency in both modes is $f_{c i}=6 \mathrm{kHz}$ larger than the frequency rotation of the $m=1$ mode, but smaller than the one of the $m=2$ mode.

For $m=2$ mode, ions are ejected between the arms and rotate with them at a velocity lower than the wave rotation velocity. But for $m=1$ mode, ions are radially ejected inside the arm, while on the edge of the structure the ionic fluxes are azimuthally oriented inward. The phase shift between the azimuthal velocity and the density perturbation is null for the $m=2$ mode, but close to $\pi / 5$ for the $m=1$ mode. Finally, no axial perturbation is present for the $m=2$ mode, whereas it is observed for the $m=1$ mode with an ion flux always directed through the collecting grid.

Centrifugal, Kelvin-Helmholtz and drift wave instabilities can play an important role in bounded magnetized plasmas. ${ }^{19,20}$ The first two ones are flute like instabilities, contradictory to our observation of axial perturbation by LIF. The third one has a theoretical radial velocity shift with respect to density equal to $\pi / 2$, which is not in agreement with our experimental results (Fig. 5). Consequently, our results show a complex plasma physics which is not fully explained by current linear theories.

\section{DISCUSSION AND SUMMARY}

The time-resolved LIF diagnostic shows that the physics underlying $m=1$ and $m=2$ rotating instabilities is strongly different. The boundary conditions of the plasma column (grids polarisations and the presence of two half-cylinders in the $m=1$ case) play an important role in the balance of the incoming/outcoming fluxes of charged particles, leading to different physics. As in the $m=2$ study, ${ }^{5}$ the naive image of a plasma exhibiting a global rotation is invalidated by our results but in a different way. Modified Simon-Hoh type instabilities $^{21-23}$ are suspected to be at the root of the 
observed $m=1$ structure, but the physics seems to be much more complicated.

Annaratone ${ }^{10}$ proposed an analytical model to reproduce the pressure and magnetic field dependence of the rotation frequency of the $m=1$ unstable modes in MISTRAL. This simplified model takes into account the radial variations of the radial ion velocity obtained by LIF measurements and is in global agreement with our results.

Recently, Pierre ${ }^{24}$ proposed another simple theoretical analysis of the rotation frequency of the instability in MISTRAL. He considered that the angular frequency of the drifting ions determines the rotation frequency of the $m=1$ mode, taking into account the effect of ion-neutral collisions on the $\vec{E} \times \vec{B}$ ionic velocity. ${ }^{25}$ But as indicated by this author, this model strongly depends on the value of the cross section of ion-neutral collisions, which is not known with enough accuracy.

Kinetic simulations by Boeuf ${ }^{14}$ have shown the possible role of the critical ionization velocity (CIV) in the $\vec{E} \times \vec{B}$ rotation of a plasma column. This could be compatible with our experimental results, even though the rotation frequency at $\mathrm{r}=6 \mathrm{~cm}$ corresponding to the $\operatorname{argon} \mathrm{CIV}(8700 \mathrm{~m} / \mathrm{s})$ is more than 4 times larger than our experimental results.

For a better understanding of the complex physics revealed by the time-resolved LIF diagnostics, there is now a strong need for more detailed numerical simulations. Our results could be used for a careful analysis of the underlying physics.

\section{ACKNOWLEDGMENTS}

The authors would like to acknowledge fruitful discussions with J-P. Boeuf and S. Mazouffre. The results presented here are part of Cyril Rebont's Ph.D. thesis.

${ }^{1}$ T. Pierre, E. Escarguel, D. Guyomarc'h, R. Barni, and C. Riccardi, Phys. Rev. Lett. 92, 065004 (2004).

${ }^{2}$ M. Matsukuma, T. Pierre, A. Escarguel, D. Guyomarc'h, G. Leclert, F. Brochard, E. Gravier, and Y. Kawai, Phys. Lett. A 314, 163 (2003).

${ }^{3}$ S. Jaeger, T. Pierre, and C. Rebont, Phys. Plasmas 16, 022304 (2009).

${ }^{4}$ A. Escarguel, Eur. J. Phys. D 56, 209-214 (2010).

${ }^{5}$ C. Rebont, N. Claire, T. Pierre, and F. Doveil, Phys. Rev. Lett. 106, 225006 (2011).

${ }^{6}$ D. N. Hill, S. Fornaca, and M. G. Wickham, Rev. Sci. Instrum. 54, 309 (1983).

${ }^{7}$ N. Claire, M. Dindelegan, G. Bachet, and F. Skiff, Rev. Sci. Instrum. 72, $4372(2001)$.

${ }^{8}$ E. I. Dobrokhotov, A. V. Zharinov, I. N. Moskalev, and D. P. Petrov, Nucl. Fusion 9, 143 (1969).

${ }^{9}$ M. Kono and M. Y. Tanaka, Phys. Rev. Lett. 84, 4369 (2000).

${ }^{10}$ B. M. Annaratone, A. Escarguel, T. Lefèvre, C. Rebont, N. Claire, and F. Doveil, Phys. Plasmas 18, 032108 (2011).

${ }^{11}$ C. Schröder, O. Grulke, and T. Klinger, Phys. Plasmas 11, 4249 (2004).

${ }^{12}$ G. Y. Antar, J. H. Yu, and G. Tynan, Phys. Plasmas 14, 022301 (2007).

${ }^{13}$ Y. N. Yeliseyev, Plasma Phys. Rep. 32, 927 (2006).

${ }^{14}$ J.-P. Boeuf and B. Chaudhury, Phys. Rev. Lett. 111, 155005 (2013).

${ }^{15}$ N. Claire and G. Bachet, Phys. Plasmas 13, 062103 (2006).

${ }^{16}$ B. Pélissier and N. Sadeghi, Rev. Sci. Instrum. 67, 3405 (1996).

${ }^{17}$ D. L. Jassby, Phys. Fluid 15, 1590 (1972).

${ }^{18}$ R. Barni, R. Riccardi, T. Pierre, A. Escarguel, D. Guyomarc'h, and K. Quotb, New J. Phys. 7, 225 (2005).

${ }^{19}$ W. Horton, Rev. Mod. Phys. 71, 735 (1999).

${ }^{20} \mathrm{~F}$. Brochard, E. Gravier, and G. Bonhomme, Phys. Plasmas 12, 062104 (2005).

${ }^{21}$ A. Simon, Phys. Fluids 6, 382 (1963).

${ }^{22}$ F. C. Hoh, Phys. Fluids 6, 1184 (1963).

${ }^{23}$ M. N. Rosenbluth and A. Simon, Phys. Fluids 8, 1300 (1965).

${ }^{24}$ T. Pierre, Phys. Plasmas 23, 042110 (2016).

${ }^{25}$ F. F. Chen, Introduction to Plasma Physics and Controlled Fusion (Plenum Press, New York, 1974). 